\title{
Lapurdum
}

LAPURDUM Euskal ikerketen aldizkaria | Revue d'études basques |

Revista de estudios vascos | Basque studies review

$15 \mid 2011$

Numéro XV

\section{Hots Ahostunak Euskal Hiztegian}

\section{Aritz Irurtzun}

URL : http://journals.openedition.org/lapurdum/2332

DOI : 10.4000/lapurdum.2332

ISSN : 1965-0655

Éditeur

IKER

Édition imprimée

Date de publication : 1 octobre 2011

Pagination : 101-141

ISSN : 1273-3830

Référence électronique

Aritz Irurtzun, « Hots Ahostunak Euskal Hiztegian », Lapurdum [Linean], 15 | 2011, Sarean emana----an 15 octobre 2014, kontsultatu 19 avril 2019. URL : http://journals.openedition.org/lapurdum/2332

DOI : 10.4000/lapurdum.2332 


\title{
Hots Ahostunak Euskal Hiztegian ${ }^{1}$
}

\author{
Aritz IRURTZUN \\ CNRS-IKER (UMR 5478)
}

Intonazioaren inguruko ikerketetan, arazo metodologiko nagusi bat izaten da galdeketetarako corpusa osatzean, izan ere, corpusa ezaugarri fonetiko-fonologiko hertsien arabera eraiki behar da uhinaren oinarrizko maiztasuna (F0, Hertz-etan neurtzen dena) jaso ahal izateko. Behaztopa-harri nagusia fonema ahoskabeen presentzia izaten da, fonazioa etetean, F0 uhina eteten da eta etenak irauten duen milisegundoetan ezin da neurketarik egin. Areago, mikroprosodiaren eraginez arazoa ez da etenetara mugatzen, eta etenaren osteko fonazioan uhinak etenik izango ez balitz izango ez lituzkeen balioak izaten ditu (nabarmenki, leherkarien osteko balioak igo egiten dira cf., i.a., Hanson (2009)). Hori dela-eta, laborategiko fonologia-ikerketak egiten dira intonazioan, hizketa libreak, oro har, zarata esperimental handia izaten baitu eta honenbestez, ez dituelako eskaintzen konparaziorako egokiak diren datuak. Horretarako, soinu ahostunez osatutako galdetegiak behar izaten dira, eta batzuetan zaila gertatzen da horiek egiteko hitz egokiak aurkitzea. Honela bada, jarraian datorrena intonazioaren ikerketan baliagarri izan daitekeen corpus bat da, soilik ahostunak diren soinuez osaturikoa.

Corpusa osatzeko Xuxen hiztegia arakatu dut eta soilik ahostunez osatutako hitzak erauzi (horretarako Unix eta PERL script pare bat baliatu ditut). ${ }^{2} 80.778$ hitzez osatutako corpusetik 5.443 hitz erauzi dira; behean ematen ditut intonazioaren ikerketarako galdetegien prestaketan lagungarri izan daitezkeelakoan.

Azkenik, corpusaren osaketaren inguruko ohar batzuk:

(i) "j" letrarekin osatutako hitzak corpusean gorde dira, euskalki batzuetan hurbilkari palatal ahostun gisa ahoskatzen delako.

(ii) Dardarkari anizkunak dituzten forma guztiak baztertu dira (hasierakoak (radar, rugby), bokalartekoak (agorril, harri), baita ere muta cum liquida atake konplexuetakoak (deabru, greba), zein coda posiziokoak arno, ardi etab.). Hitz amaierako dardarkariak dituzten hitzen kasuan, ondoren bokal bat izanik dardarkari soila izan dezaketenak corpusean

1. Lan hau ondoko dirulaguntzei esker burutu da: GIC07/144-IT-210-07 (EJ), 2011 JSH2 0041 (ANR), FFI2011-29218, (MICINN), FFI2012-38064-C02-01 (MINECO), BCS-1147083 (NSF), HiTeDi, UFI11/14 (UPV/EHU). Eskerrik asko Maia Duguine eta Gorka Elordietari haien iruzkinengatik.

2. PERLen inguruan ikasi nahi izanez gero begiratu bat eman Astigarraga-eta (2009) lanari. Hemen oinarritzat erabili den hiztegia haiek berek baliatutakoa da. 
gorde dira (nor, ur), gainontzekoak corpusetik at utzi dira.

(iii) Leherkari ahostunak benetako leherkari izan daitezkeen kasuekin bestela jokatu dut; coda osteko atakeetan daudenean (abelburu, andere) corpusean mantentzea erabaki dut. Kontua da, haietan neurketak egiteko ingurune egokirik eskaintzen ez badute ere (uhina moztera iristen direlako), neurketen inguruko testuinguruak sortzeko egokiak izan daitezkeela esperimentu batzuetan. Nolanahi ere, optimoak ez diren item hauek erraz identifikatzeko, hitzok letra etzanez agertzen dira.

(iv) Atake konplexuetako albokariak dituzten formak ere jaso dira (ganglio, doble). Hauek ere letra etzanez ematen dira. 


\section{Hiztegiko corpus ahostuna:}

A

a

Aaiun

Aaron

$\mathrm{AB}$

Abad

abade

abadegai

Abadi

abadi

abadia

abagune

abai

abail

abaila

abaildu

abaildura

abal

abala

abaldun

abalemaile

Abando

abandona

abandonu

abao

abaro

abdomen

abdominal

Abe

abe

abegi

Abel

abelbide

abelburu

abeldun

abelera

abemaria

abenda

abendu

abendualdi

abeni

abere

aberi

aberia

Aberin

Abi

abia

abiabide

\begin{tabular}{|c|c|}
\hline abiaburu & adei \\
\hline abiada & adeigabe \\
\hline abiadan & Adela \\
\hline abiadura & adela \\
\hline abiagailu & Adelaida \\
\hline abiagune & Adelina \\
\hline abialdi & adelu \\
\hline abiamen & Aden \\
\hline abian & adi \\
\hline Abidjan & adia \\
\hline Abijan & adiagile \\
\hline abil & adib \\
\hline abilamendu & adibide \\
\hline abildade & adiera \\
\hline abildu & adigabe \\
\hline abilidade & adigai \\
\hline Abilio & adiguri \\
\hline abio & adila \\
\hline abioi & adimen \\
\hline abl & adimendu \\
\hline aboa & adimendun \\
\hline aboli & adimengabe \\
\hline abona & adin \\
\hline abonamendu & adina \\
\hline abonu & adinaldi \\
\hline aborigen & adinamia \\
\hline aborin & adinaro \\
\hline Abu & adindu \\
\hline abu & adinduenagana \\
\hline Abuja & adindun \\
\hline abuli & adinean \\
\hline abulia & adinera \\
\hline Abundio & adingabe \\
\hline aburu & adinon \\
\hline ad & adio \\
\hline adaba & adj \\
\hline adabegi & adlg \\
\hline adaburu & ADN \\
\hline adagio & Adoain \\
\hline adaje & adoba \\
\hline Adam & adobe \\
\hline Adan & adoi \\
\hline Adana & adondo \\
\hline adaro & adora \\
\hline $\mathrm{adb}$ & adore \\
\hline egi & adoregabe \\
\hline FGUUI & adu \\
\hline
\end{tabular}

abiaburu

adeigabe

Adela

adela

adelu

Aden

adia

adiagile

adib

adigai

adiguri

adila

adimengabe

adin

adinamia

adinaro

indu

adinduenagana

dindun

adinean

adinera

adingabe

non

adlg

ADN

Adoain

adoba

adondo

adora

adoregabe 
aduana

aduanari

Aduna

AEB

Aedo

aerobio

aerolabangailu

aerologia

aga

agabe

Agamemnon

Ageda

Agen

agenda

agenen

ageri

agerian

agerira

ageriro

agi

agian

agidanean

agigu

agilando

agin

Aginaga

Aginagalde

agindu

aginean

agiri

aglomera

ago

agoa

agoila

agon

agondu

agonia

agoran

Aguado

Aguayo

agudo

Agueda

Aguilera

Aguinaga

Aguinagalde

agunda

Agurain agure

ah

ahabi

ahabia

ahago

ahaide

ahaidego

ahaideria

ahaire

ahal

ahala

ahalara

ahalbide

ahalbidera

ahaldun

ahaldundu

ahaldungai

ahalean

ahalegin

ahalegina

ahalegindu

ahaleginean

ahalera

ahalgabe

ahalgo

ahalmen

ahalmendu

ahalmendun

ahalorodun

ahamen

ahamenda

ahari

Ahedo

aheri

ahi

ahidura

aho

ahobero

ahoeri

ahohandi

ahomen

ahora

ahu

ahul

ahulaldi

ahuldade

ahuldu ahuldura

ahulenarena

ahuleria

ahulgune

ahulune

AI

$\mathrm{Ai}$

ai

Aia

Aiala

Aiara

aiari

aiba

aida

aidun

aiduru

Aiegi

aiei

aiena

aiene

aiengana

aieru

aihen

aihenondo

aiho

aila

ailara

ailaragin

aile

ailega

ailegabide

aimara

Ain

ainada

Ainara

ainara

ainba

ainbana

Aingeru

aingeru

aingira

aingura

ainguragile

Ainhoa

Ainoa

ainube

ainuri 


\begin{tabular}{|c|c|c|}
\hline Aio & Alava & aldamenera \\
\hline aio & Alba & aldamiaia \\
\hline Aiora & alba & aldamio \\
\hline aira & albagela & Aldana \\
\hline airaldi & albain & aldara \\
\hline aire & albaindu & aldare \\
\hline airean & albainu & aldarera \\
\hline airebide & Albania & aldaro \\
\hline airera & albaniera & aldauri \\
\hline aiui & albara & Alday \\
\hline aiuma & albaran & alde \\
\hline aiuri & albedo & aldean \\
\hline Aja & albeni & aldebana \\
\hline ajaja & albenia & aldehido \\
\hline aje & albeolo & alden \\
\hline ajea & alberjinia & aldendu \\
\hline ajeume & Albi & aldenduagoa \\
\hline Ajuria & Albina & aldengailu \\
\hline $\mathrm{Al}$ & Albino & aldera \\
\hline al & albino & alderago \\
\hline Ala & albo & aldi \\
\hline ala & albodi & aldian \\
\hline Alaba & albora & aldioro \\
\hline alaba & alborada & aldiri \\
\hline alabadere & alborengo & aldiro \\
\hline alabaduna & album & aldiune \\
\hline alabaina & albumen & Aldude \\
\hline Alabama & albumina & Aldundi \\
\hline aladonea & albuminoide & aldundi \\
\hline aladura & Alda & ale \\
\hline alaen & alda & alea \\
\hline alaena & aldaaldi & alega \\
\hline alagailu & Aldaba & alegera \\
\hline alagune & aldaba & alegi \\
\hline alagura & aldabera & Alegia \\
\hline alai & aldabide & alegia \\
\hline alaialdi & aldadenbora & alegialari \\
\hline Alain & aldaera & alegilari \\
\hline alain & aldagabe & alegoria \\
\hline alaja & aldagai & alehun \\
\hline alajaina & aldagailu & alejale \\
\hline alaldi & aldagela & Alejo \\
\hline alamen & aldagoi & alelo \\
\hline Alangoa & aldagune & aleluia \\
\hline alano & Aldai & aleman \\
\hline alara & aldaira & Alemania \\
\hline alarau & aldamen & Alen \\
\hline
\end{tabular}


aleroi

alga

algara

Algeria

alha

alhadura

alhagia

alhagune

alhan

alia

alibi

aliena

alimale

Aljeria

alla

Allah

Allende

allenen

Alli

Allin

Allo

almadia

almeja

almena

Almeria

almidoi

almoada

almohade

almorabide

Almudena

Alo

alo

aloe

aloger

alogera

aloi

aloimendu

alojamendu

alondegi

Aloria

alu

alubioi

alumina

aluminio

Alvarado

Alvaro

ama amabi

amabirjina

Amable

Amada

Amadeo

Amado

amagabe

amagai

amagoi

Amagoia

amai

Amaia

amaidin

amaidun

amaiera

amaigabe

amaina

amainari

amainu

Amalain

amalgama

Amalia

Amalloa

amama

amameru

Amanda

amanda

Amando

Amann

Amara

amara

amaraun

Amaya

ameba

ameboide

Amelia

amelu

amen

amenga

amengu

amharera

ami

Amiano

amida

amigdala

amigo

amil amila

amilbide

amilburu

amildegi

amildu

amilera

amilla

Amillaga

Amillao

amiloide

amina

amio

amira

amiral

amiralgo

amirun

Amman

Amo

amodio

amoina

amon

amona

amondu

amonio

amora

amoral

amore

amu

amugile

amulu

Amundarain

Ana

Anabel

anaerobio

anai

anaia

anaidi

anaienaren

analogia

analogo

anana

anbiguo

anbo

anboi

anbulo

anburu

and 


\begin{tabular}{|c|c|c|}
\hline Anda & Angelu & Araguaia \\
\hline & angelu & Araia \\
\hline andabide & angina & Aral \\
\hline andaburu & angio & aralde \\
\hline Andagoia & anglo & araldi \\
\hline andalan & Angola & Arama \\
\hline andana & angula & arama \\
\hline andanada & Angulema & aramagu \\
\hline andare & Ania & Aramaio \\
\hline andari & Anibal & araman \\
\hline anddere & anil & arameera \\
\hline Ande & anilina & Aramendia \\
\hline andea & anima & Arami \\
\hline andegamendu & animali & aramu \\
\hline andel & animalia & Aran \\
\hline anderauren & animo & aran \\
\hline Andere & anioi & Arana \\
\hline andere & Anjel & arana \\
\hline andi & anoa & Aranburu \\
\hline Andia & anodal & Aranda \\
\hline andiago & anodino & arandi \\
\hline Andima & anodo & Arandigoien \\
\hline Andoain & anomalia & arandoi \\
\hline andoil & anomalo & Aranguren \\
\hline andoila & anonimia & Arano \\
\hline Andoin & anonimo & aranondo \\
\hline Andolin & anu & arao \\
\hline Andollu & Anue & araolari \\
\hline Andonegi & anuro & araondo \\
\hline Andonegui & ANV & arau \\
\hline Andoni & ara & araubide \\
\hline andu & Araba & araudi \\
\hline andui & araba & araudun \\
\hline andura & arabego & arauemaile \\
\hline Ane & arabera & araugabe \\
\hline anega & araberan & araugailu \\
\hline anelido & Arabia & araugilea \\
\hline anemia & arabiera & araugileen \\
\hline anemona & arabil & Araujo \\
\hline anere & arabilgu & are \\
\hline aneroide & arabilna & area \\
\hline Angara & arada & areago \\
\hline angarila & aradol & arean \\
\hline angarilari & aragi & Arellano \\
\hline Angel & aragoera & Arenal \\
\hline angela & Aragoi & Arenala \\
\hline Angelina & aragoiera & Arenao \\
\hline
\end{tabular}


augurio

Arene

arenga

arengana

arerio

Arevalo

Arhane

ari

Aria

aria

arian

Ariane

Aribe

aribide

arie

ariel

arigu

aril

arima

arimadun

arimaduna

arimagabe

arin

arinago

arindu

arindura

aringailu

aringalari

ario

Arion

aro

Aroa

aroa

aroagu

aroan

aroi

arol

aroma

$\mathrm{Au}$

Aube

aube

Aude

aude

audio

audiologia

augura

augure auhen

auhenda

auhendari

Aulo

auma

aura

Aurea

Aurelia

Aureliano

Aurelio

aureola

auria

auriga

aurio

aurora

Avelina

Avelino

Aveyron

Avignon

Avila

Aviv

Ayala

Ayo

b

$\mathrm{Ba}$

ba

baba

babalore

Babel

babil

Babilonia

bable

babo

babuino

bada

badere

badi

badia

baga

bagaia

bagaieria

bagaje

Bagdad

bagil

bagina

bagoi
Baguia

baha

Bahama

bahe

bahegile

bahero

bahi

bahimena

bahimendu

bahola

Bahrain

Bahrein

bahuvrihi

bai

baia

baibaina

baiduria

baila

bailara

baile

bailebilen

bailegoen

baileuden

bailien

bailio

bailira

bailiran

bailiren

bailuan

bailuen

baimen

baimendu

baimendun

baimengabe

baina

bainadore

bainagia

bainera

bainila

baino

bainoago

bainu

bainugela

bainulari

Baiona

baionaren

baira 


\begin{tabular}{|c|c|c|}
\hline baja & bananondo & bari \\
\hline baje & banbu & baria \\
\hline Bajo & banda & Bariain \\
\hline bala & bandadunen & Barinaga \\
\hline balada & bandalo & Barindano \\
\hline balbe & bandalu & bario \\
\hline Balbina & bandeja & baroi \\
\hline Balbino & bandera & Baroja \\
\hline balbula & banderadun & Barola \\
\hline balda & banderari & Barona \\
\hline baldan & banderila & Bavaria \\
\hline balde & banderilero & Bayon \\
\hline baldi & banderilla & BBB \\
\hline baldin & banderillero & $\mathrm{Be}$ \\
\hline baldinba & bandidaia & be \\
\hline baldinbere & bandidu & beaba \\
\hline baldo & bando & Bearin \\
\hline Baldomero & bandolero & Beballea \\
\hline Balduin & Bandung & beberin \\
\hline bale & baneria & bebil \\
\hline balea & Bangi & beda \\
\hline balen & banilla & Bedaio \\
\hline Balendin & banimendu & bedaio \\
\hline Bali & banjo & Bedaroa \\
\hline balia & Banjul & bedel \\
\hline baliabide & bano & bedera \\
\hline baliamendu & bao & bederen \\
\hline balin & Bara & bedi \\
\hline balio & bara & Bedia \\
\hline baliodun & baragailu & Bedo \\
\hline baliogabe & baraila & beduino \\
\hline Ballariain & Barajuen & bee \\
\hline baloi & baramendu & begi \\
\hline balonbolea & Baranbio & begidun \\
\hline balora & baranda & begie \\
\hline balore & barandaila & begigu \\
\hline baloredun & Barandalla & begihandi \\
\hline BAME & Barandiaran & begin \\
\hline bamen & barano & begio \\
\hline bana & baranu & begira \\
\hline banadio & barau & begirada \\
\hline banal & baraualdi & begiragailu \\
\hline banaloria & bare & begiraile \\
\hline banan & barealdi & begiraldi \\
\hline banana & barema & begirale \\
\hline bananadi & baremo & begiralego \\
\hline banandu & bareune & begiramen \\
\hline
\end{tabular}


begiramendu

begirari

begirune

Bego

bego

begonia

beha

behari

Behaune

behe

behealde

behegain

behera

beherago

beheragune

beheraldi

beheramendu

behere

beherengo

Beherobia

behi

behiala

behin

behinean

behinen

behingo

behingoan

behinola

Behobia

Beijin

Beijing

beila

beilari

beilegi

beira

beiradura

beiragile

Beire

beirineria

Bejarano

bejon

bejondeiala

bejondeiela

bejondeigula

bejondeinala

bejondeiola

bela

belagile

\begin{tabular}{|c|c|}
\hline belagin & Bera \\
\hline belagoi & bera \\
\hline Belagua & berabil \\
\hline belai & beraiei \\
\hline belamendu & berain \\
\hline belanda & berama \\
\hline Belandia & Beramendi \\
\hline belarjale & beran \\
\hline belaun & berandu \\
\hline belaunaldi & beranduan \\
\hline belaunburu & Berango \\
\hline belaundu & Berano \\
\hline belberin & berarenaren \\
\hline bele & berau \\
\hline Belen & berauei \\
\hline belen & bere \\
\hline belena & berearen \\
\hline Belere & berearena \\
\hline belgundari & bereen \\
\hline belladona & beregain \\
\hline belladonna & beregana \\
\hline Bello & berehala \\
\hline bello & berein \\
\hline belo & beren \\
\hline belu & berendia \\
\hline beluri & berendu \\
\hline bema & berengain \\
\hline bemol & berengana \\
\hline $\mathrm{BEN}$ & berenjena \\
\hline ben & berera \\
\hline bena & Beriain \\
\hline benda & beribil \\
\hline bene & berie \\
\hline beneno & berigu \\
\hline benera & berilio \\
\hline benereo & berilo \\
\hline benga & berin \\
\hline Bengala & berina \\
\hline bengala & berinalari \\
\hline bengalera & Bering \\
\hline benial & berio \\
\hline Benigna & berjabe \\
\hline Benigno & berjera \\
\hline Benilde & bero \\
\hline Benin & beroa \\
\hline Benjamin & beroaldi \\
\hline beno & beroango \\
\hline
\end{tabular}


berogailu

berogailudun

beroi

beronen

beronena

berori

beroriei

berun

berundegi

berundun

berunduna

beruneria

beude

$\mathrm{BH}$

BI

bi

Bia

biahore

Biana

bianda

biao

biba

bibalbio

biberoi

Bibiana

bibli

Biblia

biblia

bibliog

bibora

bid

bida

bidagin

bidagiro

bidai

bidaia

bidaiari

bidaide

bidal

bidalagiri

bidaldi

bidali

Bidania

bidari

bidaro

bide

bidegabe

\begin{tabular}{|c|c|}
\hline Bidegain & bilbe \\
\hline bidegile & Bilbo \\
\hline bidegileen & bild \\
\hline bidegileon & bildil \\
\hline bidegileren & bildu \\
\hline bidegin & bilduagoa \\
\hline Bidegoian & bilduma \\
\hline bidelagun & bildumagile \\
\hline bideo & bildumari \\
\hline bideogile & bildumen \\
\hline bideogilea & bildura \\
\hline bideogileari & bilera \\
\hline bideogileen & bilgia \\
\hline bideolan & bilgu \\
\hline bidera & bilguma \\
\hline bidoi & bilgune \\
\hline bidun & bili \\
\hline biela & bilin \\
\hline bienal & bilioi \\
\hline Bienvenida & Billabona \\
\hline Bienvenido & Billela \\
\hline biera & bilo \\
\hline biga & biloba \\
\hline bigai & Biloda \\
\hline bigamia & Biloria \\
\hline bigamo & bina \\
\hline biganderia & binan \\
\hline bigira & binbi \\
\hline bigun & binbili \\
\hline bigunaldi & Bingen \\
\hline bigundu & bingo \\
\hline bigundura & binomio \\
\hline Biguria & bio \\
\hline biharamun & biola \\
\hline biharamunago & biolin \\
\hline bihi & biologi \\
\hline bihoa & biologia \\
\hline bijilia & biologo \\
\hline bil & bionbo \\
\hline bila & bira \\
\hline bilalde & biradera \\
\hline bilaldi & biragune \\
\hline Bilanane & biraldi \\
\hline bilania & birao \\
\hline bilari & biraogile \\
\hline bilau & biraolari \\
\hline bilba & birau \\
\hline
\end{tabular}


biribil

biribildu

biribilgu

biribilgune

birjina

birjindade

birudi

birunda

birundan

Bladi

blai

blenda

blinda

blindaje

BM

BN

BNG

bo

boa

boalda

bobeda

bobido

Bobigny

bobina

bobo

boda

bodega

bodegoi

BOE

Boeing

boga

bogada

bogalari

Bohemia

bol

bola

bolada

boladun

bolaluma

bolandera

bolanjeria

bolari

bolba

bolbora

bolea

boleibol

bolero boli

Bolibia

bolido

bolina

Bolivia

bolo

Bologna

bologna

bolu

bolumen

bon

bona

bonba

Bonbai

bonbilla

bonbo

bonboi

bonbona

bondade

Bonilla

Bonn

bono

bonu

boom

boreal

Borja

boro

Boroa

borobil

borobildu

borobildun

boronde

Boveda

$\mathrm{Bu}$

budin

bueno

buha

buhada

buhame

buhameria

buhunba

buia

buila

Bujan

Bujanda

bujia

Bujumbura bulba

bulbo

bulda

bulegari

bulego

Bulgaria

bulgariera

bulimia

bulo

bulunba

bulunbari

bumeran

bunbada

buril

burio

buru

Buruaga

burualde

buruarenaren

buruarin

burubero

burubide

burubio

burudun

buruera

burugabe

burugain

burugin

burugo

buruhandi

buruil

burujabe

burujale

burulan

burumena

burumenaren

burumenari

burumin

burumuin

burundara

Burundi

burura

d

da

Dabid

dabil

dabilela 


\begin{tabular}{|c|c|c|}
\hline dadi & darabil & degie \\
\hline dadila & darabilgu & degieala \\
\hline dado & darabilna & degiegu \\
\hline daga & darama & degiela \\
\hline dagenil & daramagu & degien \\
\hline dagi & daraman & degienala \\
\hline dagigu & Daria & degigu \\
\hline dagin & darie & degiguala \\
\hline dago & dariera & degigula \\
\hline dagoen & darigu & degigun \\
\hline dagoenarena & darin & degigunala \\
\hline dagoenarengan & Dario & degin \\
\hline dagola & dario & deginagu \\
\hline dagonenean & daroa & deginala \\
\hline dagonil & daroagu & degio \\
\hline dailari & daroan & degioala \\
\hline dailu & daude & degiogu \\
\hline Daily & daudela & degiola \\
\hline dalai & daudenena & degion \\
\hline dalan & daudenenari & degionala \\
\hline daldara & daudenengan & dei \\
\hline dali & daudenengana & Deia \\
\hline dalia & Dauhah & deialdi \\
\hline Dallo & daunb & deiera \\
\hline dama & David & deigailu \\
\hline Damian & Davila & deiune \\
\hline Damiana & de & dejada \\
\hline damna & Deba & del \\
\hline damu & debadio & delega \\
\hline damuari & Debagoiena & Delhi \\
\hline damubide & debalde & Delia \\
\hline dan & debalua & delibera \\
\hline danba & debeia & deliberamendu \\
\hline danbada & debeiu & delibero \\
\hline danbolin & debil & delinea \\
\hline danbolindari & deboila & dema \\
\hline Danborenea & deboiladura & demadan \\
\hline danda & deboilamendu & demagogia \\
\hline dandy & debora & demagogo \\
\hline Danel & dedu & demagun \\
\hline danga & degenera & demanda \\
\hline dangada & degiagu & demo \\
\hline dangel & degiala & demonio \\
\hline Daniel & degidaala & den \\
\hline Daniela & degidala & dena \\
\hline daniera & degidan & denarena \\
\hline Danubio & degidanala & denarengan \\
\hline
\end{tabular}




\begin{tabular}{|c|c|c|}
\hline $\begin{array}{l}\text { denbora } \\
\text { denboragailu }\end{array}$ & $\begin{array}{l}\text { Digne } \\
\text { digno }\end{array}$ & $\begin{array}{l}\text { DIU } \\
\text { divehiera }\end{array}$ \\
\hline denboral & digu & Divina \\
\hline denboraldi & digun & $\mathrm{dm}$ \\
\hline denboraldu & diharu & DNA \\
\hline denborale & Dijon & do \\
\hline denda & dilema & doa \\
\hline dendari & dilin & doala \\
\hline dendel & dilinda & doan \\
\hline dendu & dilindan & doanarena \\
\hline denena & dilubio & doba \\
\hline denengan & dilui & Dobaran \\
\hline denganino & Dima & dobela \\
\hline denge & din & dobla \\
\hline denominadore & dina & doble \\
\hline denona & dinagu & dobloi \\
\hline denongan & dinamo & Dodoma \\
\hline deriba & dinbi & dogma \\
\hline Derio & dinbili & doha \\
\hline deroga & dinda & dohain \\
\hline deun & dio & dohaindu \\
\hline deunga & diodo & doi \\
\hline di & Diodoro & doinu \\
\hline diadema & diogu & dola \\
\hline diagonal & diogunona & dolamen \\
\hline diagu & Dion & dolare \\
\hline dial & dion & Dolly \\
\hline dialogo & dira & dolmen \\
\hline dialogogileen & dirau & dolora \\
\hline Diana & diraugu & dolore \\
\hline diana & diraun & dolu \\
\hline Diario & direnena & dolumen \\
\hline dibaga & direnenean & dolumin \\
\hline diban & direnenei & domai \\
\hline dibidendu & direnenen & domaia \\
\hline dibidi & direnengan & Dome \\
\hline dibinal & diro & domeina \\
\hline dibino & dirogu & domeinu \\
\hline dibulga & diron & domina \\
\hline didan & diru & Dominga \\
\hline Didio & dirualdi & Domingo \\
\hline die & dirubide & domino \\
\hline Diego & dirudi & domu \\
\hline diegu & dirudigu & Don \\
\hline dien & dirudin & dona \\
\hline dienena & dirudun & donabera \\
\hline digeri & dirugabe & donada \\
\hline
\end{tabular}




donado
Donamaria
donari
donario
done
Donemiliaga
dongaro
donge
dongero
Donibane
donu
dora
Dorinda
dorio
doru
du
dual
Dublin
duda
dudagabe
dudaldi
dudan
duelu
duenarena
duenarengan
duenarengana
duenarenganaino
duende
duenena
duenenean
Duero
dugoin
dugu
dugunona
dugunongan
Duhalde
duin
duindu
duingabe
dun
duna
dunba
dunbada
duo

\begin{tabular}{|c|c|}
\hline duodeno & edema \\
\hline duodenu & Eden \\
\hline Dura & eden \\
\hline duraluminio & edendu \\
\hline Duran & edil \\
\hline Durana & edin \\
\hline Durangaldea & ediren \\
\hline Durango & EDL \\
\hline duro & Edmund \\
\hline durunda & Edmundo \\
\hline durundi & Edmuno \\
\hline durundidun & edo \\
\hline durundu & edonola \\
\hline dvandva & edonon \\
\hline DVD & edonongoaren \\
\hline Dvina & edonor \\
\hline DYA & edonora \\
\hline E & edu \\
\hline e & edun \\
\hline EA & EE \\
\hline $\mathrm{Ea}$ & EEA \\
\hline ea & EEE \\
\hline EAB & EG \\
\hline EAE & eg \\
\hline EAJ & EGA \\
\hline ean & Ega \\
\hline $\mathrm{EB}$ & ega \\
\hline ebain & egalda \\
\hline ebalua & egari \\
\hline ebanjelari & Egeo \\
\hline ebanjelio & EGG \\
\hline ebano & EGI \\
\hline EBB & egi \\
\hline EBO & Egia \\
\hline ed & egia \\
\hline eda & egiadun \\
\hline edabana & egiagu \\
\hline edabe & egidan \\
\hline edade & egie \\
\hline edagale & egiegu \\
\hline edale & egien \\
\hline edan & egigu \\
\hline edanaldi & egigun \\
\hline edaran & Egiguren \\
\hline edari & egile \\
\hline EDBL & egilegai \\
\hline Edelmira & Egin \\
\hline
\end{tabular}




\begin{tabular}{|c|c|c|}
\hline egin & EHAA & Elduain \\
\hline eginagu & EHAE & ELE \\
\hline eginahal & ehaile & ele \\
\hline eginahalean & Ehari & elea \\
\hline eginbide & ЕНBO & elealdi \\
\hline eginen & EHE & elebidun \\
\hline egingo & ehe & eledun \\
\hline Egino & eheina & elegia \\
\hline egio & ehelega & Elejalde \\
\hline egiogu & eheren & elemenia \\
\hline egion & eherena & Elena \\
\hline egiramu & eheri & Elene \\
\hline egiune & EHGAM & elge \\
\hline EGLU & EHME & Elgea \\
\hline ego & EHNE & Elguero \\
\hline egoaida & eho & eli \\
\hline egoaira & ehogailu & Elia \\
\hline egoal & EHU & elia \\
\hline egoera & ehule & elian \\
\hline egoile & ehun & elimina \\
\hline egon & ehunburu & Elio \\
\hline egonaldi & ehundegi & elion \\
\hline egonean & ehundu & Elmu \\
\hline egongela & ehundura & elmugara \\
\hline egongia & ehunen & Elo \\
\hline egongune & ehungailu & Eloi \\
\hline eguen & EI & Eloina \\
\hline Eguia & ei & Elomendi \\
\hline Eguiguren & eiara & Eloy \\
\hline Eguino & EIE & Elvira \\
\hline egun & eihera & EMA \\
\hline egunaldi & eiheramen & ema \\
\hline egunaria & einera & emadan \\
\hline egunariari & Eire & emagaldu \\
\hline egunbira & EJ & emagin \\
\hline egundaino & el & emagun \\
\hline egundi & ELA & emai \\
\hline egundo & elabora & emaien \\
\hline egundu & Eladia & emaile \\
\hline egunean & Eladio & emaion \\
\hline eguneango & Elagabalo & emaldi \\
\hline egunera & elai & emalege \\
\hline egunero & elaire & eman \\
\hline egungo & ELB & emanaldi \\
\hline egunoro & Elba & emandegi \\
\hline eguraldi & elbi & emangailua \\
\hline $\mathrm{EH}$ & elbidera & Emanuel \\
\hline
\end{tabular}




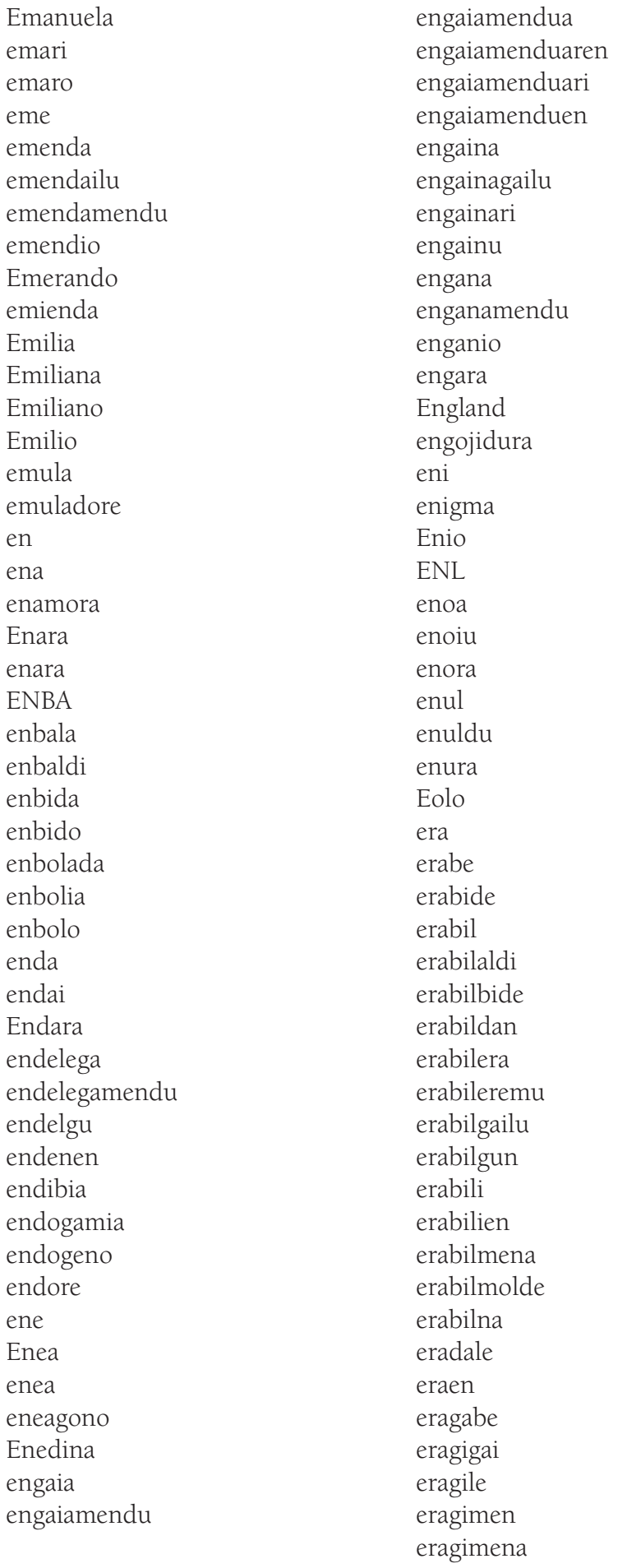

eragin

eraginbera

eragindura

eragingailu

eragole

eragon

erail

eraile

eralda

eraldi

eralgi

eramadan

eramagun

eramaien

eramaile

eramaion

eraman

Erandio

Erango

erara

Eraul

Ere

ere

ereda

eredu

eredugile

eregi

eregu

ereiaro

ereile

erein

ereinaldi

ereingailu

ereingarai

ereingiro

eremu

Erevan

eri

erialdi

Eribe

eribera

eridele

erion

eriondo

ero

eroaldi

eroale 


\begin{tabular}{|c|c|c|}
\hline eroan & gabi & gajo \\
\hline erogeno & gabial & GAL \\
\hline erogoa & gabila & gal \\
\hline eromen & Gabina & Gala \\
\hline eromena & Gabino & gala \\
\hline eromenaren & gabirai & galai \\
\hline eromenari & Gabiria & Galain \\
\hline eromenean & Gabon & galandi \\
\hline eromenera & gabon & galani \\
\hline eronde & gabonil & galania \\
\hline eror & Gaborone & Galba \\
\hline eroraldi & gadolinio & galbahe \\
\hline erori & gaelera & galbera \\
\hline erorialdi & gaeliera & galbide \\
\hline eruge & Gago & galbidera \\
\hline Erundina & gahe & galbihi \\
\hline Euba & gai & galburu \\
\hline EUDEL & gaiago & galda \\
\hline Eudel & gaila & galdaldi \\
\hline Eudima & gailego & galdara \\
\hline Eugenia & gailen & galdaragile \\
\hline Eugenio & gailendu & galdareria \\
\hline Eugi & gailu & galde \\
\hline Eulalia & gaimel & Galdeano \\
\hline euli & gaimena & galdegai \\
\hline eulihandi & gain & galdegile \\
\hline Eulogia & gainalde & galdegin \\
\hline Eulogio & gainbalio & galdera \\
\hline Eure & gainbegira & galdo \\
\hline eure & gainbegirale & galdu \\
\hline euregana & gainbehera & gale \\
\hline euri & gainbeheraldi & galeban \\
\hline eurialdi & gainbide & galegada \\
\hline euro & gaindegi & galego \\
\hline euroarenaren & gaindi & galena \\
\hline Eurogune & gaindidura & Galeno \\
\hline Euroliga & Gaindola & galeoi \\
\hline Eva & gaindu & galera \\
\hline Evangelina & gainean & galerada \\
\hline Evelia & gainera & galeradun \\
\hline Evelio & gaingabe & galeria \\
\hline Evry & gaingaila & galeriano \\
\hline Eyre & gaingari & Galerio \\
\hline$g$ & gaingora & galga \\
\hline $\mathrm{Ga}$ & gaingune & galgara \\
\hline Gabadi & gainune & galgarau \\
\hline gabai & Gaio & \\
\hline gabe & gaje & \\
\hline
\end{tabular}


Galia

galibo

Galieno

galiera

Galilea

galin

Galindo

galio

gallaldi

Gallego

Gallo

galmen

galmendi

Galo

galoi

galondo

Galvan

gama

Gambia

Gamboa

gamelu

Gamere

Gaminde

gamma

Gamue

ganadu

ganba

ganbara

Ganbe

ganbela

ganbelu

ganbera

ganbil

Ganboa

Gandhi

Gandia

gando

gandola

gandu

ganga

gangadun

gangaila

gangailen

gangarabil

gangeri

gangil

ganglio

ganora ganoradun

ganoragabe

ganu

Gara

gara

garabi

garabide

Garai

garai

garaiera

garailari

garaile

garaino

Garaio

Garaioa

garaje

garamola

garan

garandu

garau

Garay

garenengana

garenona

garenongan

gari

Garibai

garidi

garil

Garin

Garinda

Garindaine

Garinoain

gario

Garo

garo

Garoa

garoera

Garona

garondo

garun

garundegi

gau

gaualde

gaualdi

gauaro

Gaubea

gaubeila gaubele

gaude

gaudela

gauden

gaudenengan

gaudi

gaudimen

gauenara

gauero

gaugiro

gaulan

gaulehen

Gauna

gay

GB

GBB

ge

Gebara

geben

geheli

gehi

gehiago

gehiagoan

gehiegi

gehien

gehienean

gehiengo

gehiengodun

gehiengoduna

gehiengodunaren

gehiengodunen

gehigailu

geide

geina

gel

gela

gelagune

gelari

gelaune

gelba

gelbera

geldi

geldiago

geldialdi

geldiera

geldiro

geldiune 


\begin{tabular}{|c|c|c|}
\hline Geldo & geninan & geunde \\
\hline geldo & genio & geundean \\
\hline gelge & genioan & geunden \\
\hline Gelio & genioen & geundenan \\
\hline Gellao & genion & geundu \\
\hline Gema & genionan & geure \\
\hline Gemini & genirau & geurearen \\
\hline Gemma & genirauan & geureen \\
\hline Genara & genirauen & geuregana \\
\hline Genbe & geniraunan & geuron \\
\hline Gendulain & geniro & Ghana \\
\hline gene & geniroen & gi \\
\hline genealogia & genirudi & gia \\
\hline genegi & genirudian & gibel \\
\hline genegian & genirudien & gibela \\
\hline genegie & genirudinan & gibelalde \\
\hline genegiean & Genoa & gibelaldi \\
\hline genegien & Genobeba & gibelamendu \\
\hline genegienan & genoma & gibelari \\
\hline genegin & Genoveva & gibelera \\
\hline geneginan & genu & gibeljale \\
\hline genegio & genuen & gibelmin \\
\hline genegioan & genuenona & gibelondo \\
\hline genegion & geogenia & gibelori \\
\hline genegionan & geoide & giboi \\
\hline generabil & geologia & gida \\
\hline generabilean & geologo & gidabaimen \\
\hline generabilen & geonomia & gidagailu \\
\hline generabilenan & gera & gidaila \\
\hline generadore & geraldi & gidaliburu \\
\hline generama & geranio & gidari \\
\hline generamaan & Gere & gidoi \\
\hline generaman & Gerea & gidoigile \\
\hline generamanan & Gerediaga & gidoilari \\
\hline genero & gereino & gidoin \\
\hline generoa & geren & giga \\
\hline generoaan & Gerendiain & Gijon \\
\hline generoan & geri & Gil \\
\hline generoanan & gero & gila \\
\hline generodun & geroago & gilbera \\
\hline Geneva & geroaldi & Gilen \\
\hline genian & geroenean & giligili \\
\hline genie & gerora & Gillen \\
\hline geniean & gerorengo & gilori \\
\hline genien & gerundio & Gimeno \\
\hline genienan & geu & gin \\
\hline genin & geun & gina \\
\hline
\end{tabular}


ginbail

ginbaildun

ginda

Gindano

Ginea

ginen

ginenona

ginga

gingil

gingondo

gino

ginoan

giralda

Giraldelli

giranoi

giri

giro

girogailu

Girona

Gironda

gladiadore

glande

glandula

gleba

global

globo

globulina

globulu

Gloria

gloria

gnomo

Go

goaia

goal

goalada

gobada

Gobeo

gobio

Godavari

godo

Godoy

godu

Goenaga

goga

gogabera

gogai

gogaide

gogaiduraren gogaiera

Goiri

gogaldi

Goiuri

gogalgin

gogamen

gogara

gogo

gogoa

gogoango

gogoarin

gogobera

gogogabe

gogoilun

gogolari

gogonduri

gogora

gogoramen

gogoramendu

goha

gohain

gohaindi

gohaindidura

gohaindu

gohaindura

goi

Goiain

goiaingeru

goialde

Goialdea

goialdi

goianengo

Goiballea

goibehe

goibehera

goibel

goibelaldi

goibeldu

goibeldura

Goiburu

goiburu

goien

goiende

goiengo

goiera

goihabe

goihendura

goinahi

Goio
Gojenola

gol

gola

Golan

golda

golde

goldelari

golegile

Golem

Gollano

golo

goma

gomadun

gomagabe

gomenda

gomendio

gomendu

Gomera

gona

gonada

gonadun

gonagai

gonagain

gonbida

gonburu

gondola

gondolari

gong

gongoil

Gongora

gora

gorabehera

gorabide

gorabidera

goragale

gorago

goragune

goraldi

goramen

goranahi

Gorane

goraune

Gore

goren

gorenarenaren 


\begin{tabular}{|c|c|c|}
\hline gorendu & gureen & hain \\
\hline gorengo & guregain & Hainandao \\
\hline gori & guregana & hainba \\
\hline gorila & guren & hainbana \\
\hline goroldi & gurenda & hala \\
\hline goroldio & gurendu & halere \\
\hline goru & guri & hall \\
\hline gorulari & gurin & halo \\
\hline gorule & gurindegi & halogeno \\
\hline gorune & guriro & haluro \\
\hline gu & guru & hamabi \\
\hline Guadalajara & guruin & hamabiren \\
\hline Guadiana & Guyana & hamahiru \\
\hline guai & $\mathrm{h}$ & hamahiruren \\
\hline guaia & habail & hamalau \\
\hline guaiaba & habaila & hamalaudun \\
\hline guaiabondo & habailari & han \\
\hline guajiro & habaildu & handi \\
\hline Guam & Habana & handiago \\
\hline guano & habanera & handiagoarena \\
\hline guarani & HABE & handidun \\
\hline guaraniera & habe & handiduna \\
\hline guau & habi & handienarena \\
\hline gubia & habia & handiera \\
\hline guda & habil & handigai \\
\hline gudalburu & habilela & handigailu \\
\hline gudaldi & haboro & handigoa \\
\hline gudari & habuin & handigura \\
\hline gudu & hadi & handinahi \\
\hline gudulari & hadila & handiro \\
\hline Gue & HAEE & hangoaren \\
\hline guen & Haga & hangoen \\
\hline guena & haga & Hanibal \\
\hline gugana & hagin & Hanoi \\
\hline Guggenheim & haginlari & hara \\
\hline guhaur & hago & harabil \\
\hline Guido & hagoa & harabilgu \\
\hline Guinea & hagoadura & haragi \\
\hline gula & hagoan & haragidun \\
\hline Gulia & hagola & haragijale \\
\hline gun & hagoran & harago \\
\hline gune & hagorandu & haraindi \\
\hline gura & hagun & harama \\
\hline guramen & hagunda & haramagu \\
\hline gurari & haia & haran \\
\hline gure & haiei & Harana \\
\hline gurearen & haiengana & Harare \\
\hline
\end{tabular}




\begin{tabular}{|c|c|c|}
\hline harea & hegalada & helduera \\
\hline haren & hegalaldi & heldugabe \\
\hline harenga & hegalari & heldugailu \\
\hline harengana & hegalda & heldura \\
\hline hari & hegaldari & Helena \\
\hline haridun & hegaldi & helgabe \\
\hline harigai & hegaldun & helio \\
\hline harigile & hegan & Heliodora \\
\hline haril & heganagi & Heliodoro \\
\hline harildu & hegemoni & helmen \\
\hline harjo & hegemonia & helmenean \\
\hline haro & hegi & helmuga \\
\hline haroa & hegidan & helmugara \\
\hline haroagu & hegie & hemen \\
\hline haroren & hegien & hemengo \\
\hline harori & hegigo & hemoglobina \\
\hline hau & hegigoa & henbil \\
\hline hauei & hegigu & henbilen \\
\hline haugu & hegigun & Hendaia \\
\hline hauma & hegio & hendi \\
\hline $\mathrm{HB}$ & hegion & hendila \\
\hline heavy & hego & hendin \\
\hline hebain & hegoaida & hengo \\
\hline hebaindu & hegoaira & hengoen \\
\hline hebaindura & hegoalde & $\mathrm{HEO}$ \\
\hline heda & hegoburu & hera \\
\hline hedabana & hegodun & herabe \\
\hline hedabide & hegogabe & herabil \\
\hline hedadura & heia & herabilen \\
\hline hedagai & heiagora & heraldo \\
\hline hedagailu & heiara & herama \\
\hline hedagi & hein & heraman \\
\hline hedagune & heinera & Here \\
\hline hedailo & hejira & hereda \\
\hline edaldi & hel & heredero \\
\hline hedamen & hela & Heredia \\
\hline hede & helaro & hereje \\
\hline edegile & helbide & heren \\
\hline hedegileen & helbidera & herenamona \\
\hline hederia & helbideragabe & herenegun \\
\hline heg & helburu & herenegungoaren \\
\hline ega & helde & hereniloba \\
\hline egabera & helderia & Herio \\
\hline hegada & heldu & herio \\
\hline hegadun & heldualdi & heroa \\
\hline gagailu & helduaro & heroan \\
\hline gal & heldubada & heroi \\
\hline
\end{tabular}




\begin{tabular}{|c|c|c|}
\hline heroienen & hilgura & hiri \\
\hline heroina & hilobi & hirialde \\
\hline heroinomano & hilobira & hiribide \\
\hline Heron & hilon & hiribildu \\
\hline herori & hilondu & Hiriburu \\
\hline heu & hiloro & hiriburu \\
\hline heure & Himalaia & hiriduna \\
\hline heuregana & Himalaya & hirigile \\
\hline $\mathrm{HI}$ & himen & hirigin \\
\hline hi & himno & hirigune \\
\hline hidalgia & hinderabil & hiringuru \\
\hline hidalgo & hinderabildan & hiro \\
\hline hidan & hinderabilen & hirodura \\
\hline hie & hinderabilgu & hiroen \\
\hline hien & hinderabilgun & hiru \\
\hline hiena & hinderama & hirudi \\
\hline Hieron & hinderamadan & hirudien \\
\hline higa & hinderamagu & hirudun \\
\hline higadura & hinderamagun & hiruhilero \\
\hline higana & hinderaman & hirur \\
\hline higi & hinderoa & hirurehun \\
\hline higialdi & hinderoadan & hirurogei \\
\hline higibera & hinderoagu & hirurogeiren \\
\hline higidura & hinderoagun & hoa \\
\hline higiene & hinderoan & hoala \\
\hline higigailu & hindi & hobari \\
\hline higigune & hindiera & hobe \\
\hline higimen & hindoa & hobeago \\
\hline Higinia & hindoan & hobealdi \\
\hline Higinio & hindu & hoben \\
\hline higu & hindudan & hobendari \\
\hline higuin & hinduen & hobendi \\
\hline higuinaldi & hindugu & hobendun \\
\hline higuindu & hindugun & hobenduri \\
\hline higuindura & hio & hobengabe \\
\hline higun & hioen & hoberen \\
\hline hihaur & hioide & hoberena \\
\hline hil & hion & hoberendu \\
\hline hilaldi & hira & hobi \\
\hline Hilaria & hiradura & hobira \\
\hline Hilario & hiramen & hobiragabe \\
\hline hildu & hiramendu & Hodei \\
\hline hilduma & hirau & hodei \\
\hline hildura & hirauen & hodi \\
\hline hile & hire & hodiduna \\
\hline hilero & hiregain & hodieria \\
\hline hilgai & hiregana & hodira \\
\hline
\end{tabular}




\begin{tabular}{|c|c|c|}
\hline hogei & horiei & ibilaldi \\
\hline hogeiren & hu & ibilbide \\
\hline Hogi & hudigo & ibildaun \\
\hline hola & hudo & ibilera \\
\hline Holanda & huelga & ibilga \\
\hline Holgado & Huelva & ibilgailu \\
\hline holmio & huen & ibilge \\
\hline Holy & Hugo & ibilgu \\
\hline Homero & huia & ibilgune \\
\hline homilia & hule & ibilgura \\
\hline hominido & Human & ibili \\
\hline homogamia & humano & ibilian \\
\hline homogeneo & Humaran & iboil \\
\hline homologa & humero & Ibon \\
\hline homologia & humore & Ibone \\
\hline homologo & Hungaria & id \\
\hline homonimia & hungariera & Idaho \\
\hline homonimo & hur & idan \\
\hline hona & hura & ideal \\
\hline honaindi & Huron & idei \\
\hline honaindian & I & ideia \\
\hline honaino & i & ideiadun \\
\hline honda & IA & ideial \\
\hline hondabide & ia & ideialdu \\
\hline hondalan & iagu & ideologi \\
\hline hondamen & iaio & ideologia \\
\hline hondamendi & ianbo & ideologo \\
\hline hondamendira & IB & idi \\
\hline hondea & Iba & idigai \\
\hline hondeagailu & Ibai & idilio \\
\hline hondo & ibai & idioma \\
\hline hondogabe & Ibaia & Idoi \\
\hline hondora & ibaibide & idoi \\
\hline honela & ibaidun & Idoia \\
\hline honen & ibaiduna & idola \\
\hline honena & ibaira & idolo \\
\hline honengan & Iban & idoro \\
\hline Hong & ibarjaun & Idoya \\
\hline honi & Iberia & idun \\
\hline Honiara & iberiera & iduri \\
\hline Honolulu & Ibero & IE \\
\hline Honorio & IBI & ie \\
\hline horail & ibi & IEE \\
\hline horaildu & ibid & iegu \\
\hline hori & ibide & iel \\
\hline horidun & ibigune & ieldu \\
\hline horidura & ibil & ien \\
\hline
\end{tabular}




\begin{tabular}{|c|c|c|}
\hline ig & ihi & iman \\
\hline iga & Iholdi & imandu \\
\hline Igaegi & ihui & imanduna \\
\hline igali & ijiji & Imanol \\
\hline igan & il & IMI \\
\hline igana & ilagin & imilaun \\
\hline igande & ilagindegi & imina \\
\hline igandero & ilaia & immola \\
\hline igara & ilaje & immune \\
\hline igaraba & ilara & immunoglobulina \\
\hline igaran & Ilari & immunologia \\
\hline igarandu & ilaun & in \\
\hline Igari & ilaundu & inagu \\
\hline igaro & ilaundun & inaugura \\
\hline igaroaldi & ilbehera & inbadi \\
\hline igarobide & ilbilo & inbidia \\
\hline igboera & ildo & indaba \\
\hline igel & ildodun & indagora \\
\hline Igeldo & ilduma & India \\
\hline igele & ildura & indiaba \\
\hline igeri & ile & Indiana \\
\hline igerialdi & iledun & indiano \\
\hline igerian & ilegabe & indibaba \\
\hline igerigailu & ilegal & indibidual \\
\hline igerilari & ilehodi & indibidualimo \\
\hline igi & ilehori & indibiduo \\
\hline iglu & ilen & indidaba \\
\hline igneo & ileon & indigena \\
\hline ignominia & ilgora & indio \\
\hline igo & iloba & indioilo \\
\hline Igoa & ilobira & indoario \\
\hline igoaldi & ilon & Indurain \\
\hline igobide & Iluminada & ine \\
\hline igoera & ilun & inga \\
\hline igogailu & ilunago & inge \\
\hline Igone & ilunalde & ingelera \\
\hline igu & ilunaldi & ingeniari \\
\hline igual & Ilundain & ingi \\
\hline iguana & ilundegi & inginiari \\
\hline iguin & ilundi & ingira \\
\hline igun & ilundu & ingude \\
\hline igurai & ilundura & inguma \\
\hline igurain & ilunenarena & ingura \\
\hline Iguria & ilungo & ingurabide \\
\hline Igurin & ilungune & inguragune \\
\hline ihabali & imajina & inguralde \\
\hline Ihaben & imajinagin & inguraldi \\
\hline
\end{tabular}




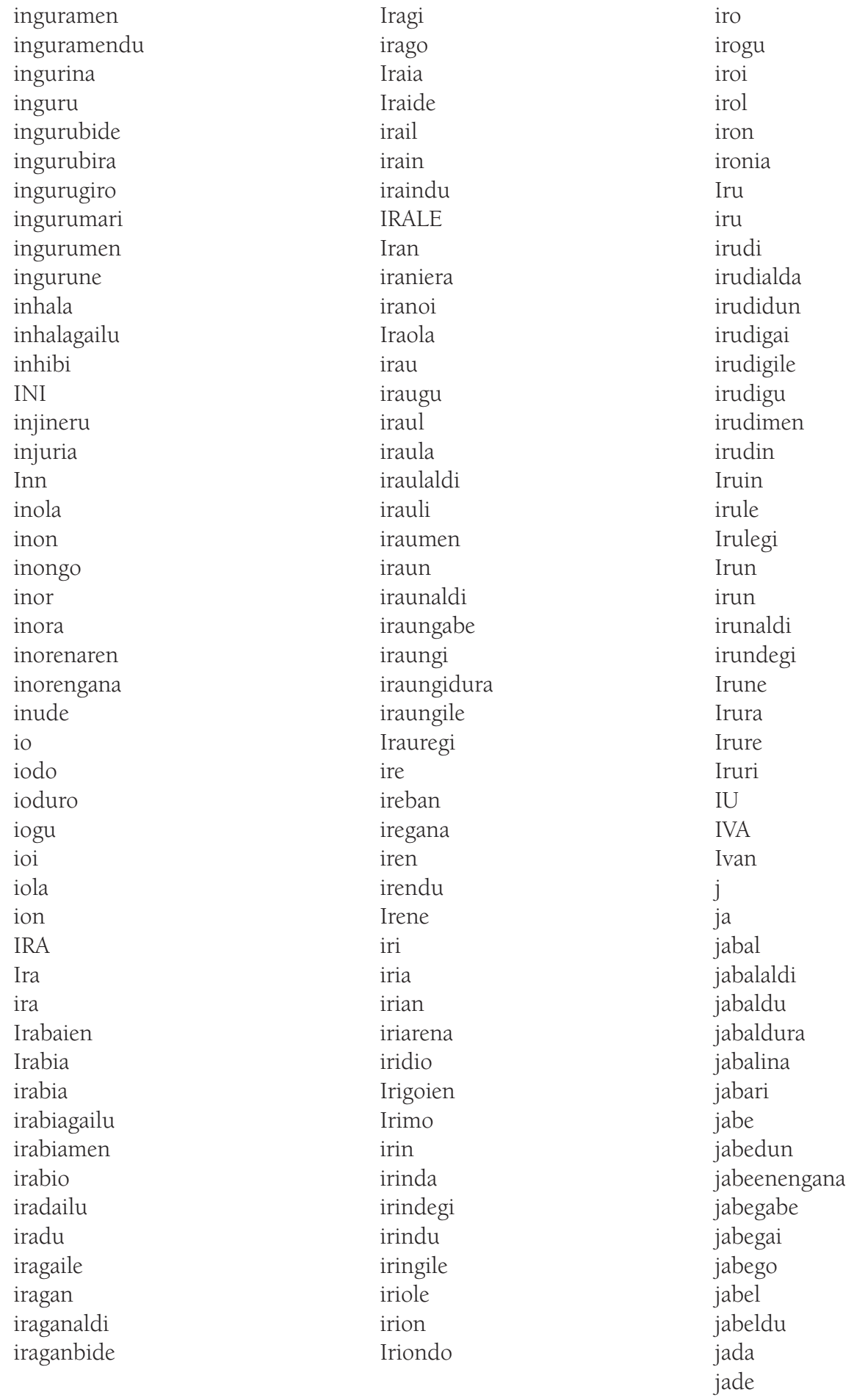


Jaen

jagi

Jagoba

jagole

jagolego

jagoleria

jagon

jagongailu

jai

jaialdi

jaiburu

jaidura

jaiegun

jaiera

jaiero

jaigai

Jaime

jaimen

Jaio

jaio

jaioera

Jaione

Jairo

jale

jalgi

jalgialdi

jalgune

Jam

jamen

jan

janaldi

janari

janba

janbo

Jandiola

jandun

Janeiro

jangai

jangale

jangela

jangura

jaramon

jare

jaregile

jaregin

jareile

jaria jariagai

jario

jarioaldi

jarioan

jaron

jarondu

jaugin

jauja

jaun

Jaunde

jaundi

jaundone

jaundu

jauneria

jaungo

jauraldi

Jauregi

jauregi

Jauregui

Java

Javi

Jayo

Jean

jean

jeina

jeinu

jela

jele

Jenara

Jenaro

jenda

jendaia

jendaila

jendalde

jende

jendearenagana

jendedi

jendilaje

jeneral

jeneraldu

jeneralean

jeneralgo

jenero

jenial

jenio

jeron

Jeronima
Jeronimo

jibaro

Jihad

Jimeno

jin

jira

jirabira

jirabiran

JJGG

jn

jo

joaera

joaira

joaldi

joaldun

joale

Joan

joan

Joana

joanaldi

Joane

joanera

joare

jod

Joel

joera

joga

jogailu

joia

joile

jole

jomuga

Jon

jondone

jondoni

Jone

joran

jori

jorubera

joule

Joviano

Juan

Juana

Juba

jubenil

jubila

jubileu

Juda 


\begin{tabular}{|c|c|c|}
\hline judegu & labeldunaren & lanari \\
\hline juderia & labendai & lanbada \\
\hline judo & labera & Lanbara \\
\hline judu & Labio & lanbide \\
\hline juglare & Laboa & Landa \\
\hline Jugo & labora & landa \\
\hline juja & laboral & landabide \\
\hline jujamendu & laborari & landagailu \\
\hline jujari & labore & landare \\
\hline juje & Ladoga & landaredi \\
\hline jujuba & lady & landaregai \\
\hline Julen & laga & landarejale \\
\hline Julene & lagi & landola \\
\hline Julia & lagin & landu \\
\hline Julian & lagindegi & landugabe \\
\hline Juliana & lagindu & landun \\
\hline Juliano & lagineria & landunen \\
\hline Julio & lagingailu & lanegun \\
\hline junbo & lagle & lanera \\
\hline June & Lago & laneremu \\
\hline jungla & lagun & langa \\
\hline Jura & lagunarenagana & langabe \\
\hline jura & lagundi & langadun \\
\hline Jurado & lagundu & langai \\
\hline Juvenal & lagunduagoa & langailu \\
\hline 1 & lagungo & Langara \\
\hline $\mathrm{La}$ & lai & langela \\
\hline la & laia & langile \\
\hline LAB & laiari & langileburu \\
\hline lab & Laida & langilego \\
\hline laba & laida & langileria \\
\hline lababo & laido & langin \\
\hline labadora & lain & langune \\
\hline Labaien & laina & Laniuri \\
\hline labain & lainadura & lanjer \\
\hline labainaldi & laino & lanolina \\
\hline labaindu & laira & Lara \\
\hline labaindura & lama & laranja \\
\hline labairu & lamada & laranjada \\
\hline labana & lami & laranjadi \\
\hline Labayru & lamia & laranjaduna \\
\hline labe & lamienaren & laranjondo \\
\hline Labeaga & lamina & lare \\
\hline labealdi & Lamindao & laringe \\
\hline label & lan & laringologia \\
\hline labeldun & lanada & laringologo \\
\hline labelduna & lanaldi & laru \\
\hline
\end{tabular}




\begin{tabular}{|c|c|c|}
\hline larumin & legegin & lema \\
\hline Larunbe & legelari & lemadun \\
\hline lau & legen & Leman \\
\hline lauburu & legenda & lemari \\
\hline lauda & legendario & Lemoa \\
\hline laudamen & legendun & Lena \\
\hline Laudio & legendundegi & lengoai \\
\hline laudo & legeria & lengoaia \\
\hline laudorio & legi & lengoaje \\
\hline laudun & legie & Lenin \\
\hline laugain & legigu & Leo \\
\hline laugune & legin & Leon \\
\hline lauoindun & legio & leon \\
\hline laur & legionario & lera \\
\hline Laura & legionella & lerabil \\
\hline Laureana & lego & lerama \\
\hline Laureano & legoa & leri \\
\hline laurehun & legumina & leria \\
\hline Laurendi & lehen & lerie \\
\hline Lauro & lehenago & lerigu \\
\hline laurogei & lehenaingeru & Lerin \\
\hline Laval & lehenaldi & lerin \\
\hline Lavin & lehenbailehen & lerio \\
\hline Lavinia & lehenengo & leroa \\
\hline le & lehengai & leude \\
\hline Lea & lehengo & leun \\
\hline Leaburu & lehengora & leunaldi \\
\hline Leagi & lehia & leundu \\
\hline Leal & lehiadun & leungailu \\
\hline lebil & lehiadura & Leyre \\
\hline led & lehiari & $\mathrm{LH}$ \\
\hline ledania & lehoi & LHL \\
\hline Ledea & lei & liana \\
\hline ledi & leial & liba \\
\hline ledila & leialdi & Libano \\
\hline lega & leiho & Libaroa \\
\hline legal & leihoburu & Libe \\
\hline legamia & leinu & libelo \\
\hline legamiadun & leinuru & libelu \\
\hline legamiagabe & Leioa & libera \\
\hline Legaria & Leire & liberal \\
\hline lege & Leiun & liberaldun \\
\hline legealdi & lela & Liberia \\
\hline legedi & lele & libero \\
\hline legegabe & lelo & Libia \\
\hline legegai & leloaldi & libido \\
\hline \multirow{2}{*}{ legegile } & lelodun & liburu \\
\hline & leloduna & liburugile \\
\hline
\end{tabular}




\begin{tabular}{|c|c|c|}
\hline Lide & linja & logale \\
\hline lidiera & linje & logela \\
\hline lie & linjeria & logia \\
\hline Lieja & Lino & logura \\
\hline liga & linoleo & lohi \\
\hline ligain & linolio & lohidura \\
\hline ligamendu & linondo & lohigabe \\
\hline Ligi & lio & Loidi \\
\hline Liginaga & lira & loina \\
\hline lignina & lirain & Loiola \\
\hline ligu & liraindu & Loira \\
\hline ligula & lirau & Loire \\
\hline liho & lirin & Loiu \\
\hline lihoa & lirindu & loja \\
\hline lihoburu & lirio & lojamendu \\
\hline lihondo & liro & lojamendua \\
\hline lila & lirudi & lolo \\
\hline lili & Livia & Lome \\
\hline Lille & Livio & lomen \\
\hline lilura & Ljubljana & lona \\
\hline lilurabide & Llaguno & London \\
\hline liluradura & llama & longain \\
\hline liluraldi & Llano & Longida \\
\hline liluramendu & Llarena & Longo \\
\hline Lima & Lleida & longo \\
\hline lima & llollo & lora \\
\hline limadura & lo & loradenda \\
\hline limagailu & loaldi & lorail \\
\hline limari & loba & loraldi \\
\hline lime & Lobamba & lore \\
\hline limnologia & lobel & Lorea \\
\hline limo & Lobo & loredi \\
\hline limoi & lobonen & loredun \\
\hline limoiondo & lobula & loreil \\
\hline limonada & lobulu & Lorena \\
\hline limondo & lodi & lorenen \\
\hline limuri & lodiago & loreneni \\
\hline $\operatorname{lin}$ & lodiera & loria \\
\hline Lina & lodigune & lorio \\
\hline linabera & lodiune & loro \\
\hline linai & lodoi & Lovaina \\
\hline linbo & LOE & LRU \\
\hline lindano & loeragile & lu \\
\hline lindo & loeragingailu & lualdi \\
\hline linea & loeri & Luanda \\
\hline lineal & loeria & luba \\
\hline lingala & logabe & lubera \\
\hline
\end{tabular}


Lubiano

ludi

Luengo

lugi

lugin

Lugo

luhumenda

luhumendu

luia

luma

lumadi

lumadun

lumagabe

lumaje

lumalda

lumera

Lumo

Luna

lunbago

lurjabe

Lydia

Lyon

$\mathrm{m}$

Ma

ma

mabi

Madalena

madalena

madari

Madaria

Madariaga

madariondo

Maddi

Madeira

madura

magal

magalo

Magdalena

magdalena

magia

magilari

magina

maginara

magingune

magma

magnolia

mago
Maguna

Maguregi

Maguregui

magurio

mahai

mahaiburu

mahaiondo

mahaira

Mahanadi

maharaja

mahi

maho

mahoi

Mahoma

mahuma

mahunga

Mai

Maia

maia

Maialen

maila

mailadi

mailega

mailegari

mailegu

mailu

mailubi

maina

mainada

maindire

maingeria

maingu

maingueri

maingueria

maiola

maioria

Maioriano

maira

mairu

majo

Majuro

malabarera

Malabo

Malaga

malaria

malba

malda
Maldiva

maldivera

Maldonado

Male

malgu

Mali

Mallabia

malma

malmai

malo

malur

Malvina

mama

mamala

mambo

Mami

mami

mamidun

mamigabe

mamu

man

mana

Manado

managailu

Managua

manaiu

Manama

manamendu

manba

manda

mandabide

mandarin

mandarina

mandarinera

mandarinondo

Mandela

mandeme

mandeuli

mandi

mandia

mandiei

mandien

mandil

mandileria

mandio

mando

Mandoiana 


\begin{tabular}{|c|c|c|}
\hline mandolina & Mari & media \\
\hline mandu & mari & mediana \\
\hline manea & Maria & Mediavilla \\
\hline maneia & maria & medien \\
\hline maneiu & Marian & Medina \\
\hline manenen & marian & medio \\
\hline manera & Mariana & medium \\
\hline maneragabe & Mariano & megalomania \\
\hline manerala & Maribel & megalomano \\
\hline Manero & marihuana & meha \\
\hline mangal & Marin & mehail \\
\hline mangel & Marina & Mehaine \\
\hline mangelo & marina & mehe \\
\hline mangera & marinada & meheduna \\
\hline mango & Marinda & mehego \\
\hline mangolino & marinel & mehegune \\
\hline mangondo & marineleria & mei \\
\hline mangune & Marino & meilora \\
\hline mani & marinonen & mejiloi \\
\hline mania & Mario & Mela \\
\hline Manila & marionen & mela \\
\hline maniura & marjina & melanina \\
\hline manjola & marjinal & meldo \\
\hline Manjon & Maro & mele \\
\hline manjura & maro & melenga \\
\hline Manoli & maruga & melenge \\
\hline Manolo & Maruri & Melero \\
\hline manonen & mau & Melida \\
\hline Manu & Maule & Melilla \\
\hline $\operatorname{manu}$ & Mauma & melinga \\
\hline manual & Maura & melodi \\
\hline Manuel & Mauro & melodia \\
\hline Manuela & Mayenne & meloi \\
\hline manugin & Mayo & Melun \\
\hline maoriera & Mbabane & Melville \\
\hline Mara & $\mathrm{Me}$ & memela \\
\hline mara & mea & memelo \\
\hline marabedi & Meabe & memo \\
\hline maradaila & Meaga & memora \\
\hline marai & meagile & memorandum \\
\hline marea & Meano & memoria \\
\hline mareabehera & MEB & memoriadun \\
\hline mareagora & medaila & memorial \\
\hline maremagnum & medailoi & memorio \\
\hline maren & medea & men \\
\hline marenen & Medellin & Menagarai \\
\hline areo & medera & menda \\
\hline
\end{tabular}




\begin{tabular}{|c|c|c|}
\hline Mendabia & mero & mimo \\
\hline Mendaro & meroera & $\min$ \\
\hline Mendavia & meru & mina \\
\hline Mende & $\mathrm{mg}$ & minbera \\
\hline mende & $\mathrm{Mi}$ & minberadura \\
\hline mendean & $\mathrm{mi}$ & Mindanao \\
\hline mendebal & mia & Mindegia \\
\hline mendebalde & Miami & mindu \\
\hline mendebaldu & miau & minduagoa \\
\hline mendel & miaulari & mindulin \\
\hline mendema & Midi & mindun \\
\hline mendemuga & Miel & mindura \\
\hline mendera & mielga & minduri \\
\hline menderagailu & mielina & mineral \\
\hline Mendi & Migel & mineraldu \\
\hline mendi & Miguel & mineralogia \\
\hline mendialde & miguru & mingabe \\
\hline Mendibe & mihauli & mingain \\
\hline Mendibil & mihi & mingila \\
\hline Mendibile & mihidun & minguru \\
\hline mendigune & mihidura & minia \\
\hline Mendiguren & mihigabe & minigona \\
\hline Mendiola & mihilu & minimo \\
\hline Mendiondo & mihimen & minimum \\
\hline mendira & mihura & minio \\
\hline Mendivil & mihuri & Mioma \\
\hline mendoi & mila & mira \\
\hline mendu & Milan & mirabe \\
\hline menera & Milano & mirail \\
\hline mengel & milaren & miramendu \\
\hline mengeldu & milenio & Miramon \\
\hline mengoa & mili & Miranda \\
\hline menia & Milia & Mirandaola \\
\hline meninge & milia & Mirande \\
\hline Menoio & milibare & Mirari \\
\hline Menoyo & milinga & mirari \\
\hline menu & milioi & mirarigile \\
\hline mera & milioidun & Mireia \\
\hline merenge & milioiduna & Miren \\
\hline meria & milioidunaren & mirenen \\
\hline Merida & milioidunena & Mireya \\
\hline meridiano & milioiren & Miriam \\
\hline merienda & Millan & Mirian \\
\hline merinalde & Milludi & Miron \\
\hline merindade & Milon & miru \\
\hline Merino & milonen & Miryam \\
\hline merio & mima & \\
\hline
\end{tabular}




\begin{tabular}{|c|c|c|}
\hline $\begin{array}{l}\text { mm } \\
\text { mobimendu }\end{array}$ & $\begin{array}{l}\text { Monge } \\
\text { mongol }\end{array}$ & $\begin{array}{l}\text { mugonean } \\
\text { muhuri }\end{array}$ \\
\hline mobla & Mongolia & muhuru \\
\hline moda & mongoliera & muila \\
\hline modal & moni & muilo \\
\hline modela & monje & muin \\
\hline modelo & monogamia & muindun \\
\hline modem & monogamo & muino \\
\hline modera & monolabore & mula \\
\hline modu & monomero & mulu \\
\hline modula & monomio & Mumio \\
\hline modulu & Mora & mumu \\
\hline Mogel & moral & muna \\
\hline mogol & Moran & Munain \\
\hline moi & Moravia & mundano \\
\hline moien & more & mundial \\
\hline moila & moredin & Mundo \\
\hline moja & morena & mundu \\
\hline mojagai & Moreno & mundualdi \\
\hline mol & Morillo & mundura \\
\hline Molano & moro & Mungia \\
\hline molda & Moroni & Muniain \\
\hline moldadura & Moya & muoi \\
\hline moldaera & Moyano & mura \\
\hline Moldava & $\mathrm{Mu}$ & muraila \\
\hline Moldavia & muda & mural \\
\hline moldaviera & mudadura & muralgile \\
\hline molde & mug & Murelu \\
\hline moldea & muga & Murillo \\
\hline moldegabe & mugaegun & murina \\
\hline moldura & mugagabe & Muro \\
\hline mole & mugagabedunen & Muru \\
\hline molibdeno & Mugairi & Murua \\
\hline Molina & mugalari & Murumendi \\
\hline Molinero & mugalde & MVLN \\
\hline Molinilla & mugaldi & Myanma \\
\hline Molinuevo & mugara & $\mathrm{n}$ \\
\hline moma & mugg & naba \\
\hline momia & mugi & nabala \\
\hline momo & mugida & nabari \\
\hline momolo & mugidalda & nabaro \\
\hline mon & mugidura & nabe \\
\hline mona & mugiera & nabiga \\
\hline monada & mugigai & nabil \\
\hline mondejo & mugimendu & nabilela \\
\hline mondoina & Mugiro & nabilen \\
\hline ondongo & mugon & nabo \\
\hline onema & Mugondo & \\
\hline
\end{tabular}


nadi

Nadia

nadila

NAEM

naga

nagi

nagialdi

nagidura

nago

nagola

nagon

Nagore

nahaba

naharo

nahi

Nahia

nahian

nahiera

nahierara

nahigabe

nahigabealdi

nahigo

nahimen

nahinon

nahinor

nahinora

Naia

naia

Naiara

nain

Nairobi

Namibia

NAN

nano

narabil

narabilen

narabilna

narama

naraman

Naranjo

nare

narea

naro

Naroa

naroa

naroan

naroro

nau naun

Nauru

nauruera

navajo

NBA

NBB

NBE

ndebelera

neba

negal

negaldun

negel

negi

negian

negie

negiean

negien

negienan

negin

neginan

negio

negioan

negion

negionan

negu

negualde

negualdi

negubide

neguburu

negugo

neguil

neguri

neguro

nehola

neholere

nehon

nehor

Neira

Nelida

nenbil

nenbilean

nenbilen

nenbilenan

nendi

nendila

nendin

nengo nengoan

nengoen

nengonan

neodimio

neoi

neol

neoliberal

neon

nerabe

nerabil

nerabilean

nerabilen

nerabilenan

nerama

neramaan

neraman

neramanan

nerau

Nere

Nerea

nereida

Nereo

neroa

neroaan

neroan

neroanan

Neron

neroni

neror

Nerva

neu

neur

neuralgia

neure

neuregana

neurologia

neurologo

neurona

Nevada

ni

nia

Niagara

Niamei

nian

nibel

nibela

nibelbide 


\begin{tabular}{|c|c|c|}
\hline nibelgailu & nirau & nongura \\
\hline nie & nirauan & nor \\
\hline niean & nirauen & Nora \\
\hline nien & niraunan & nora \\
\hline nienan & nire & norabide \\
\hline Nieva & nirearen & norabidera \\
\hline nigana & nireen & noragabe \\
\hline Nigeria & nireenean & noragiri \\
\hline nihaur & niregain & noranahi \\
\hline Nilo & niregana & noria \\
\hline $\operatorname{nin}$ & nireon & noruegiera \\
\hline ninan & niro & Norvegia \\
\hline ninbo & niroen & norvegiera \\
\hline ninderabil & nirudi & Nou \\
\hline ninderabilan & nirudian & Novo \\
\hline ninderabilean & nirudien & Novoa \\
\hline ninderabilen & nirudinan & nu \\
\hline ninderabilenan & Njamena & Nubia \\
\hline ninderabilna & no & nuen \\
\hline ninderabilnan & noa & nuenarengana \\
\hline ninderama & Noain & Nueva \\
\hline ninderamaan & noala & Nuevo \\
\hline ninderaman & noan & Nuin \\
\hline ninderamanan & nobedade & nulu \\
\hline ninderoa & Nobel & numen \\
\hline ninderoaan & nobela & numeradore \\
\hline ninderoan & nobelagile & numeral \\
\hline ninderoanan & nobelio & numerario \\
\hline nindian & nobia & numero \\
\hline nindinan & nobillada & Nuria \\
\hline nindoa & nobillero & nylon \\
\hline nindoaan & nobio & $\mathrm{O}$ \\
\hline nindoan & noble & o \\
\hline nindoanan & nodal & oa \\
\hline nindu & nodo & Oba \\
\hline ninduan & nodulu & obal \\
\hline ninduen & Noe & obala \\
\hline nindun & noel & obalo \\
\hline nindunan & nola & obario \\
\hline ninguno & nolanahi & Obdulia \\
\hline nini & nom & obe \\
\hline nio & nomada & obeago \\
\hline nioan & nomina & obedi \\
\hline niobio & nominal & oberen \\
\hline nioen & non & oberena \\
\hline nion & nonagerari & obira \\
\hline nionan & nonahi & obliga \\
\hline & nongo & obo \\
\hline
\end{tabular}




\begin{tabular}{|c|c|c|}
\hline oboe & Ohiana & oionen \\
\hline oboide & Ohiane & Ojanguren \\
\hline obula & ohidura & Ojeda \\
\hline obulaldi & ohigabe & ojiba \\
\hline obulu & ohil & Ola \\
\hline oda & ohildu & ola \\
\hline odei & ohm & Olabe \\
\hline Odiaga & $\mathrm{OHO}$ & olagara \\
\hline odira & ohoin & Olague \\
\hline odol & ohoindu & Olaia \\
\hline odolaldi & ohoingo & olajaun \\
\hline odolbero & ohol & Olalde \\
\hline odolbide & oholdegi & Olaldea \\
\hline odolbildu & oholeria & olanaren \\
\hline odoldu & ohora & Oland \\
\hline odoldun & ohore & Olano \\
\hline odolemaile & ohoredun & olanon \\
\hline odolgabe & ohoregabe & Olaran \\
\hline odolgiro & OI & olde \\
\hline odoljario & oi & ole \\
\hline odoluri & Oiane & olea \\
\hline Odon & Oianguren & olearen \\
\hline odonen & oidium & Olegario \\
\hline $\mathrm{OEH}$ & Oieregi & oleoa \\
\hline og & oihal & Olga \\
\hline ogale & oihaldu & olga \\
\hline ogen & oihaldun & olgura \\
\hline ogenduru & oihalgile & Olha \\
\hline ogengabe & oihan & Olhaibi \\
\hline ogi & Oihana & olia \\
\hline ogiba & oihanbide & oliadura \\
\hline ogibide & oihandi & oliaran \\
\hline ogibihi & oihandu & oliba \\
\hline ogidun & Oihane & olibadi \\
\hline ogigile & oihanera & olibo \\
\hline oha & oihu & olibondo \\
\hline ohagain & oihulari & olidi \\
\hline ohaide & oihumin & olio \\
\hline ohaidego & oilanda & oliobide \\
\hline ohala & oilaran & oliodura \\
\hline OHE & oilo & oliondo \\
\hline ohe & oilojale & Oliva \\
\hline oheburu & oin & Oliveri \\
\hline ohedun & oinalde & Olivia \\
\hline ohelagun & oindun & Ollaran \\
\hline ohera & oinlodi & Ollo \\
\hline ohi & Oion & Ollogoien \\
\hline
\end{tabular}




\begin{tabular}{|c|c|c|}
\hline Olmo & ongailu & ori \\
\hline olo & ongi & Oria \\
\hline Oma & ongialdi & Oribe \\
\hline Oman & ongiegi & original \\
\hline omega & ongile & orijinal \\
\hline omen & ongin & orin \\
\hline omenaldi & onginahi & Orio \\
\hline omendu & ongiro & Orive \\
\hline omniboro & ongunde & oriya \\
\hline omologa & ongura & Oro \\
\hline on & onil & oro \\
\hline Onaindia & onoidade & oroegile \\
\hline ONB & onoimen & orogenia \\
\hline onbera & ONU & oroi \\
\hline onbide & onua & oroigailu \\
\hline onbidera & onura & oroilore \\
\hline onbu & onuradun & oroimen \\
\hline onda & oogamia & orojale \\
\hline ondamendira & or & Oromi \\
\hline ondamu & ora & oron \\
\hline ondare & orail & orona \\
\hline onddo & orain & oronen \\
\hline ondea & orainago & orube \\
\hline ondina & orainaldi & OUA \\
\hline ondo & oraindaino & Ovidio \\
\hline ondoan & oraindino & Oviedo \\
\hline ondoegi & oraindio & OVNI \\
\hline Ondona & orainen & $\mathrm{u}$ \\
\hline ondonahi & orainengo & UA \\
\hline ondora & oraingo & Uagadugu \\
\hline ondore & oraingoan & Ubago \\
\hline ondorean & oraino & Ubani \\
\hline ondorego & oraldi & ubehera \\
\hline ondoren & oramahai & ubel \\
\hline ondorenean & Orange & ubeldu \\
\hline ondorengo & orano & ubeldura \\
\hline ondorio & ore & ubelune \\
\hline ondu & oregano & ubeluri \\
\hline ondugabe & Oregi & Ubera \\
\hline ondula & Oregui & ubera \\
\hline One & orein & Uberaga \\
\hline onean & Oreja & UBG \\
\hline Onega & Orella & UBI \\
\hline onenarena & oremahai & Ubide \\
\hline onenena & oren & ubide \\
\hline onenenean & Orendain & ubil \\
\hline onera & Ori & uda \\
\hline
\end{tabular}




\begin{tabular}{|c|c|c|}
\hline Udabe & uhindu & Unamuno \\
\hline udagara & uhindura & Unanu \\
\hline udagoien & uhoilo & Unanua \\
\hline udal & uhol & Unanue \\
\hline Udala & uholde & unbela \\
\hline udalburu & uholdu & Undio \\
\hline udalde & uhubi & une \\
\hline udaldi & uhui & unean \\
\hline udalehen & uhuri & UNED \\
\hline udamin & uhuria & uneoro \\
\hline Udana & ui & unib \\
\hline udara & uia & unide \\
\hline udare & UIB & unio \\
\hline udareondo & Ulia & Union \\
\hline udaro & Ulloa & UNO \\
\hline UEA & ulu & unore \\
\hline UEL & ululari & ur \\
\hline $\mathrm{UEO}$ & uma & Urabain \\
\hline UEU & umagin & Uraga \\
\hline ugabere & umaldi & urahi \\
\hline ugal & umandi & Ural \\
\hline Ugalde & ume & uralde \\
\hline ugalde & umealdi & uraldi \\
\hline ugaldu & umedun & Uranga \\
\hline ugalmen & umedunaldi & uranio \\
\hline Uganda & umegile & Urano \\
\hline Ugao & umegin & urarin \\
\hline Ugaran & umel & urarindu \\
\hline Ugarana & umeldu & urea \\
\hline ugari & umidura & uremia \\
\hline ugariago & umil & uremiadun \\
\hline ugaridun & umildade & Uria \\
\hline ugariduna & umildu & Uribe \\
\hline ugu & umildura & urin \\
\hline uhaga & umilia & urinda \\
\hline uhal & umo & urindadura \\
\hline Uhalde & umore & urindaldi \\
\hline uhaldi & umoregile & Uriona \\
\hline uhaleria & umoregilea & Uriondo \\
\hline uhandi & umoregileari & uro \\
\hline uhara & un & urodelo \\
\hline uharan & una & uroilanda \\
\hline uharo & unada & uroilo \\
\hline uhe & unadura & Urola \\
\hline uhera & Unai & urologia \\
\hline uheri & unai & urologo \\
\hline uhin & unaldi & urubi \\
\hline uhindi & unama & Uruguai \\
\hline
\end{tabular}




$\begin{array}{lll}\text { Urumea } & \text { Varona } & \text { Villanueva } \\ \text { uve } & \text { vaudeville } & \text { Vivendi } \\ \text { V } & \text { Vega } & \text { Volga } \\ \text { Vadillo } & \text { Veiga } & \text { y } \\ \text { Vaiven } & \text { Vela } & \text { YA } \\ \text { Val } & \text { Veleio } & \text { Ya } \\ \text { Valbuena } & \text { vendera } & \text { Yahoo } \\ \text { Valdivia } & \text { Vera } & \text { Yanire } \\ \text { Valeriana } & \text { Vero } & \text { Yaren } \\ \text { Valeriano } & \text { Viana } & \text { Yemen } \\ \text { Valerio } & \text { Vidal } & \text { yen } \\ \text { Valero } & \text { Videgain } & \text { Yerai } \\ \text { Valladolid } & \text { Viedma } & \text { Yeray } \\ \text { Valle } & \text { Viena } & \text { yo } \\ \text { Vallejo } & \text { Vienne } & \text { yoga } \\ \text { Van } & \text { Vigo } & \text { yogi } \\ \text { vanadio } & \text { Vila } & \text { Yolanda } \\ \text { Vara } & \text { Villa } & \text { Yon } \\ \text { Varela } & \text { Villalba } & \text { Yonne } \\ \text { Varo } & \text { Villaluenga } & \text { yuan }\end{array}$

\section{Erreferentziak:}

Astigarraga, Aitzol, Koldo Gojenola, Kepa Sarasola eta Aitor Soroa, 2009, TAPE:Testuanalisirako PERL erremintak, Bilbo: UEU.

Hanson, Helen, M., 2009, "Effects of obstruent consonants on fundamental frequency at vowel onset in English", Journal of the Acoustical Society of America, 125(1): 425-441. 